\title{
Chimera and chimera-like states in populations of nonlocally coupled homogeneous and heterogeneous chemical oscillators
}

\author{
Simbarashe Nkomo
}

Mark R. Tinsley

Kenneth Showalter

\section{Digital Commons Citation}

Nkomo, Simbarashe; Tinsley, Mark R.; and Showalter, Kenneth, "Chimera and chimera-like states in populations of nonlocally coupled homogeneous and heterogeneous chemical oscillators" (2016). Faculty \& Staff Scholarship. 81.

https://researchrepository.wvu.edu/faculty_publications/81

This Article is brought to you for free and open access by The Research Repository @ WVU. It has been accepted for inclusion in Faculty \& Staff Scholarship by an authorized administrator of The Research Repository @ WVU. For more information, please contact beau.smith@mail.wvu.edu. 


\section{AIP $\mid$ chaos \\ An Interdisciplinary Journal of Nonlinear Science}

Chimera and chimera-like states in populations of nonlocally coupled homogeneous and heterogeneous chemical oscillators

Simbarashe Nkomo, Mark R. Tinsley, and Kenneth Showalter

Citation: Chaos 26, 094826 (2016); doi: 10.1063/1.4962631

View online: http://dx.doi.org/10.1063/1.4962631

View Table of Contents: http://scitation.aip.org/content/aip/journal/chaos/26/9?ver=pdfcov

Published by the AIP Publishing

\section{Articles you may be interested in}

Weak chimeras in minimal networks of coupled phase oscillators

Chaos 25, 013106 (2015); 10.1063/1.4905197

Metastability and chimera states in modular delay and pulse-coupled oscillator networks

Chaos 22, 043131 (2012); 10.1063/1.4766592

Chimeras in a network of three oscillator populations with varying network topology

Chaos 20, 043122 (2010); 10.1063/1.3499502

Chimera states in heterogeneous networks

Chaos 19, 013113 (2009); 10.1063/1.3068353

Synchronization in interacting populations of heterogeneous oscillators with time-varying coupling Chaos 18, 037114 (2008); 10.1063/1.2979693

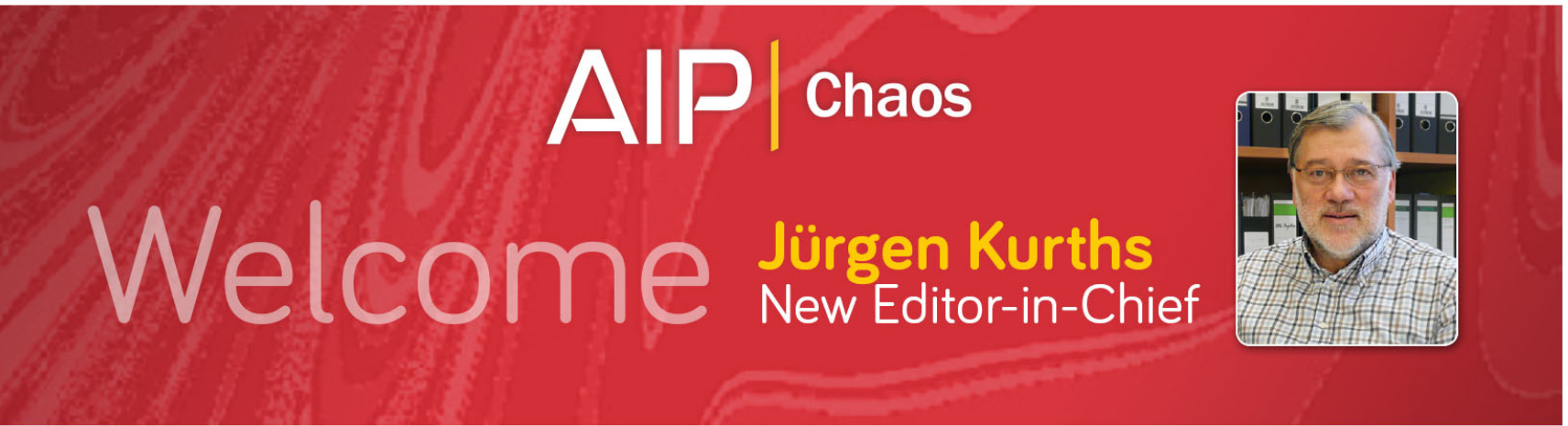




\title{
Chimera and chimera-like states in populations of nonlocally coupled homogeneous and heterogeneous chemical oscillators
}

\author{
Simbarashe Nkomo, ${ }^{1,2}$ Mark R. Tinsley, ${ }^{1}$ and Kenneth Showalter ${ }^{1}$ \\ ${ }^{1}$ C. Eugene Bennett Department of Chemistry, West Virginia University, Morgantown, \\ West Virginia 26506-6045, USA \\ ${ }^{2}$ Department of Chemistry, Bowdoin College, Brunswick, Maine 04011, USA
}

(Received 31 March 2016; accepted 26 August 2016; published online 23 September 2016)

\begin{abstract}
Chimera and chimera-like states are characterized in populations of photochemically coupled Belousov-Zhabotinsky (BZ) oscillators. Simple chimeras and chimera states with multiple and traveling phase clusters, phase-slip behavior, and chimera-like states with phase waves are described. Simulations with a realistic model of the discrete BZ system of populations of homogeneous and heterogeneous oscillators are compared with each other and with experimental behavior. Published by AIP Publishing. [http://dx.doi.org/10.1063/1.4962631]
\end{abstract}

The chimera state was first identified in a system of identical phase oscillators, in which each oscillator was coupled in an identical manner to other oscillators in its neighborhood. ${ }^{1,2}$ A number of variations on the original chimera state have been characterized, including breathing chimeras, amplitude and amplitude mediated chimeras, multiple phase-cluster chimeras, and traveling phase-cluster chimeras. ${ }^{3-9}$ In this work, we demonstrate the existence of a number of these states as well as new states in an experimental system of photochemically coupled Belousov-Zhabotinsky (BZ) oscillators, complemented by computational studies of the associated model system. We describe the similarities and differences of chimera and chimera-like states in populations of nonlocally coupled homogeneous and heterogeneous chemical oscillators.

\section{INTRODUCTION}

Since its discovery, the chimera state has been extensively investigated $^{2-18}$ (for recent review, see Ref. 19), and it has been found also to exist in systems of delay coupled and globally coupled oscillators, as well as in a variety of network structures. ${ }^{7,20-24}$ Although many theoretical studies have focused on systems of phase oscillators, chimeras have also been studied in networks with nodes that follow other dynamics, including the Ginzburg-Landau, Lorenz, Stuart-Landau, and FitzHugh-Nagumo systems. ${ }^{1,6,8,20,24,25}$

Experimental examples of chimera states were first reported in 2012 in two different systems. One developed a physical realization of a coupled map lattice, ${ }^{26}$ and the other used photochemically coupled groups of BZ chemical oscillators. ${ }^{27}$ Since then, chimera states have been observed in systems of mechanically coupled metronomes, ${ }^{28}$ electrochemical metal-dissolution oscillators, ${ }^{29,30}$ electronic frequency-modulated delay oscillators, ${ }^{31}$ electro-oxidation of silicon, ${ }^{22,32}$ and delayed-feedback semiconductor lasers. ${ }^{33}$

In this work, we further explore chimera and chimeralike states in experiments and the corresponding model system. The experiments are carried out with the photosensitive, ruthenium-catalyzed $\mathrm{BZ}$ reaction, ${ }^{34,35}$ where catalyst loaded particles in catalyst-free reaction mixtures are used to form populations of discrete chemical oscillators. ${ }^{27,36,37}$ The use of the $\mathrm{Ru}(\mathrm{bpy})_{3^{2+}}$ catalyst allows the phase of each oscillator to be perturbed with light from a spatial light modulator (SLM). The gray level $I_{j}$ of each oscillator, $j=1,2, \ldots, N$, is monitored with a CCD camera, which is used to determine the light intensity from the SLM projected on each oscillator $\phi_{j}$ according to the coupling relation

$$
\phi_{j}=\phi_{0}+\sum_{\rho=j-n}^{j+n} K\left(I_{\rho}(t-\tau)-I_{j}(t)\right),
$$

where $\phi_{0}$ is the background light intensity ${ }^{38}$ and $\tau$ is the time delay $^{20,27,37}$ in the feedback from the neighboring oscillators $\rho$ to oscillator $j$. A coupling radius of $n$ neighbors to each side of an oscillator is used with the coupling function $K=K^{\prime}$ $\exp (-\kappa|\rho-j|)$, where $K^{\prime}$ and $\kappa$ are constants that govern the effective coupling strength and range. The coupling scheme allows the oscillators to be configured on a 1-D ring with periodic boundary conditions. Further information about the experimental set-up and procedure can be found in Appendix A, Subsections 1-3.

Simulations of the nonlocally coupled chemical oscillators are carried out using the two-variable Zhabotinsky, Bucholtz, Kiyatkin, Epstein (ZBKE) model for the BZ reaction, ${ }^{39}$ modified to describe the photosensitivity of the ruthenium-catalyzed discrete oscillator system: ${ }^{27,36,37}$ $d X_{j} / d t=f\left(X_{j}, Z_{j}, q_{j}\right)+k_{s}\left(X_{j}-X_{s}\right)+\phi_{j} / \epsilon_{1}, d Z_{j} / d t=g\left(X_{j}, Z_{j}, q_{j}\right)$ $+2 \phi_{j}$, where $f$ and $g$ represent the non-photochemical components of the $\mathrm{BZ}$ reaction, and $X_{j}, Z_{j}$, and $q_{j}$ are $\left[\mathrm{HBrO}_{2}\right]$, $\left[\mathrm{Ru}(\mathrm{bpy})_{3}^{3+}\right]$, and the stoichiometric factor associated with the $j$-th oscillator. The loss of $\mathrm{HBrO}_{2}$ from oscillator $j$ to the surrounding solution is described by the term $k_{s}\left(X_{j}-X_{s}\right)$, where $k_{s}$ is the exchange constant and $X_{s}$ is the solution concentration, assumed to be constant. ${ }^{40}$ The photoexcitatory feedback to oscillator $j$ is $\phi_{j}$, calculated according to Eq. (1), where the gray levels $I_{\rho}$ and $I_{j}$ are replaced by $Z_{\rho}$ and $Z_{j}$, as the gray level is proportional to the concentration of the oxidized catalyst concentration $\left[\mathrm{Ru}(\mathrm{bpy})_{3}^{3+}\right]$. Further 
information about the model system and numerical procedures can be found in Appendix A, Subsection 4.

\section{SIMPLE CHIMERA STATES}

A chimera state in a system of oscillators with equivalent coupling can be broadly defined as a state made up of two or more coexisting subgroups exhibiting different dynamical behaviors, with one typically being incoherent in nature. The simplest chimera state observed in our simulations and experiments is composed of two groups, one with phase-locked oscillators and the other with incoherent oscillators. Examining the period distributions of the oscillators offers insights into this and other chemical oscillator chimera and chimera-like states. We also utilize a local order parameter, ${ }^{14}$ defined as $R(j, t)=\frac{1}{2 m+1}\left|\sum_{\rho=j-m}^{j+m} \exp (i \theta(\rho, t))\right|$, with $m=3$, in characterizing the spatiotemporal evolution of the chimera and chimera-like states.

Figure 1(a) shows a snapshot of a chimera state observed in our experimental system, with synchronized oscillators, $j=9-17$, coexisting with an asynchronous group, $j=1-8$ and $j=18-40$. The evolution of the synchronized group can be seen in Fig. 1(b), where the local order parameter is plotted as a function of time. The synchronized group exists for the duration of the experiment (typically $30 \mathrm{~min}$ ), with the group size fluctuating as oscillators are recruited to and released from the group.

A scatter plot of 25 consecutive values of the period of each oscillator (Fig. 1(c)) shows a broad range of periods for the incoherent oscillators. The period of the synchronized oscillators is approximately constant over the same interval of time. Figure 1(d) shows the period distributions of two unsynchronized oscillators and one synchronized oscillator. The synchronized oscillator has a shorter period and a narrow period distribution compared with the incoherent oscillators. The occurrence plot clearly identifies the two different groups associated with the chimera state.

Chimera states are observed in simulations of BZ systems with homogeneous and heterogeneous oscillator frequencies. The natural period of the homogeneous oscillators and mean period and standard deviation of the heterogeneous oscillators in our simulations are 41.0 and $41.0 \pm 2.1$, respectively. Figure 2(a) shows the local order parameter as a function of time in a system with homogeneous oscillators. Large variations in period are exhibited by the incoherent oscillators, $j=1-13$ and $j=26-30$, as shown in Fig. 2(b). Details of the coherent and incoherent oscillations are shown in the inset of Fig. 2(b), which gives the length of consecutive periods of a synchronized oscillator $(j=20$, red) and two unsynchronized oscillators ( $j=5$, magenta; $j=30$, blue). The synchronized group has a constant, shorter period, and the group exhibits distinct size fluctuations, as oscillators join and are released from the group (Fig. 2(a)). Figure 2(c) shows the local order parameter as a function of time for simulations of a BZ system with heterogeneous oscillators. Again, a synchronized region with a shorter, constant period is seen adjacent to asynchronous regions, with oscillators that have large variations in period, as shown in Figure 2(d). The length of consecutive periods of a synchronized oscillator $(j=20$, red $)$ and two unsynchronized oscillators $(j=5$, magenta; $j=30$, blue) is shown in the inset of Fig. 2(d). Size fluctuations of the synchronized group are also seen in the heterogeneous system; however, the heterogeneity gives rise to a less well defined boundary between the synchronized and asynchronous oscillators (Fig. 2(c)).

\section{CHIMERA-LIKE STATES WITH PHASE WAVES}

Figure 3 shows a type of behavior observed in our experiments and simulations with heterogeneous oscillators that we refer to as chimera-like states with phase waves.
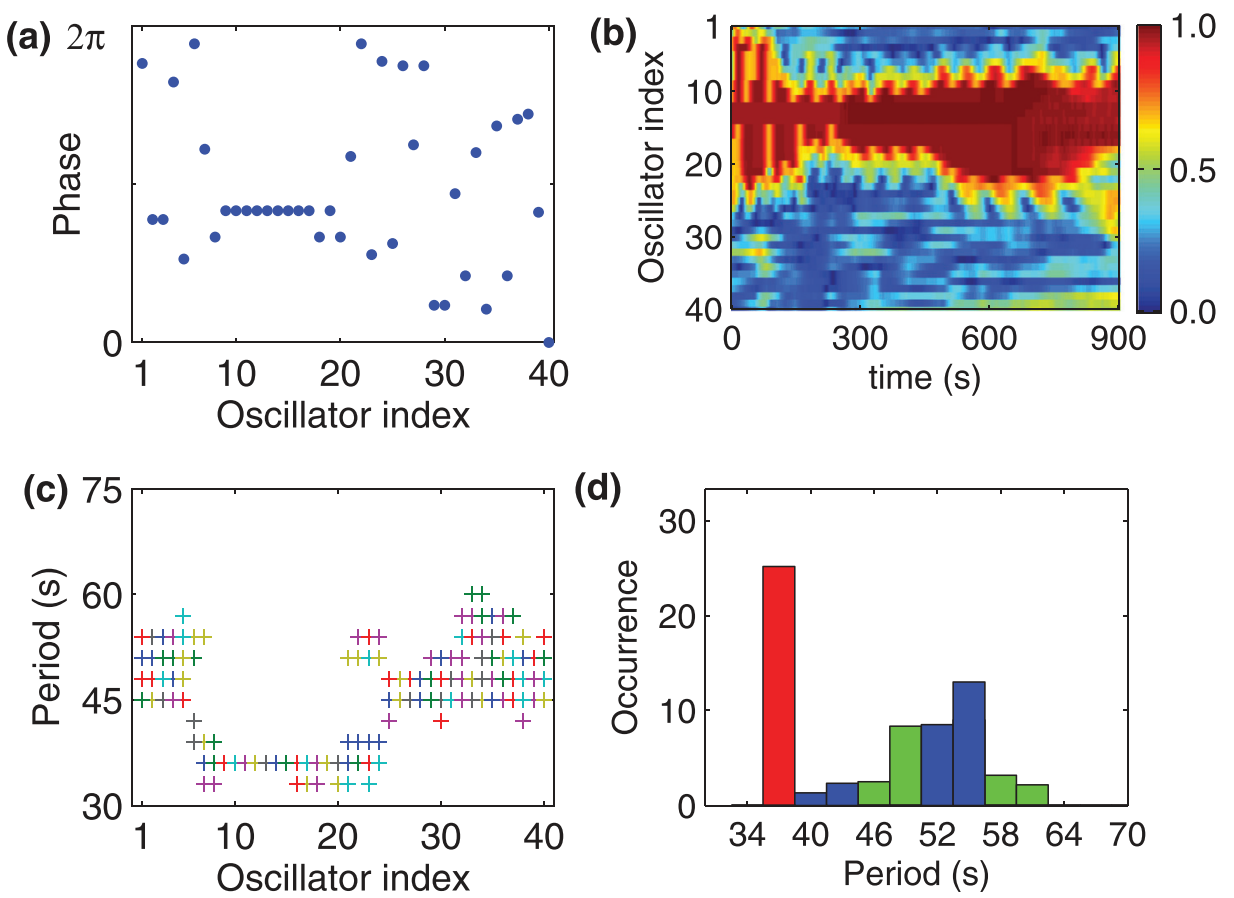

FIG. 1. Experimental measurements illustrating a simple chimera state in a system of 40 coupled BelousovZhabotinsky (BZ) oscillators. The experiment was initiated with a phase synchronized subset of the oscillators, with the remaining oscillators having an approximately random phase distribution. The nonlocal coupling was introduced at $t=300 \mathrm{~s}$. (a) A snapshot of the phase of each oscillator at $t=515 \mathrm{~s}$. (b) Space-time plot of the local order parameter, $R(j, t)=\frac{1}{2 m+1}$ $\left|\sum_{\rho=j-m}^{j+m} \exp (i \theta(\rho, t))\right|$, with $m=3$. (c) A scatter plot of 25 consecutive periods for each oscillator. (d) Occurrence plot of the periods of three oscillators, one synchronized (red) and two unsynchronized (blue, green), $j=10,6$, and 33, respectively. Experimental conditions: $\left[\mathrm{NaBrO}_{3}\right]$ $=0.51 \mathrm{M}, \quad[$ Malonic acid $]=0.16 \mathrm{M}$, $[\mathrm{NaBr}]=0.08 \mathrm{M}, \quad\left[\mathrm{H}_{2} \mathrm{SO}_{4}\right]=0.77 \mathrm{M}$, $n=10, \kappa=0.5, K^{\prime}=1.0, \tau=30 \mathrm{~s}$. 

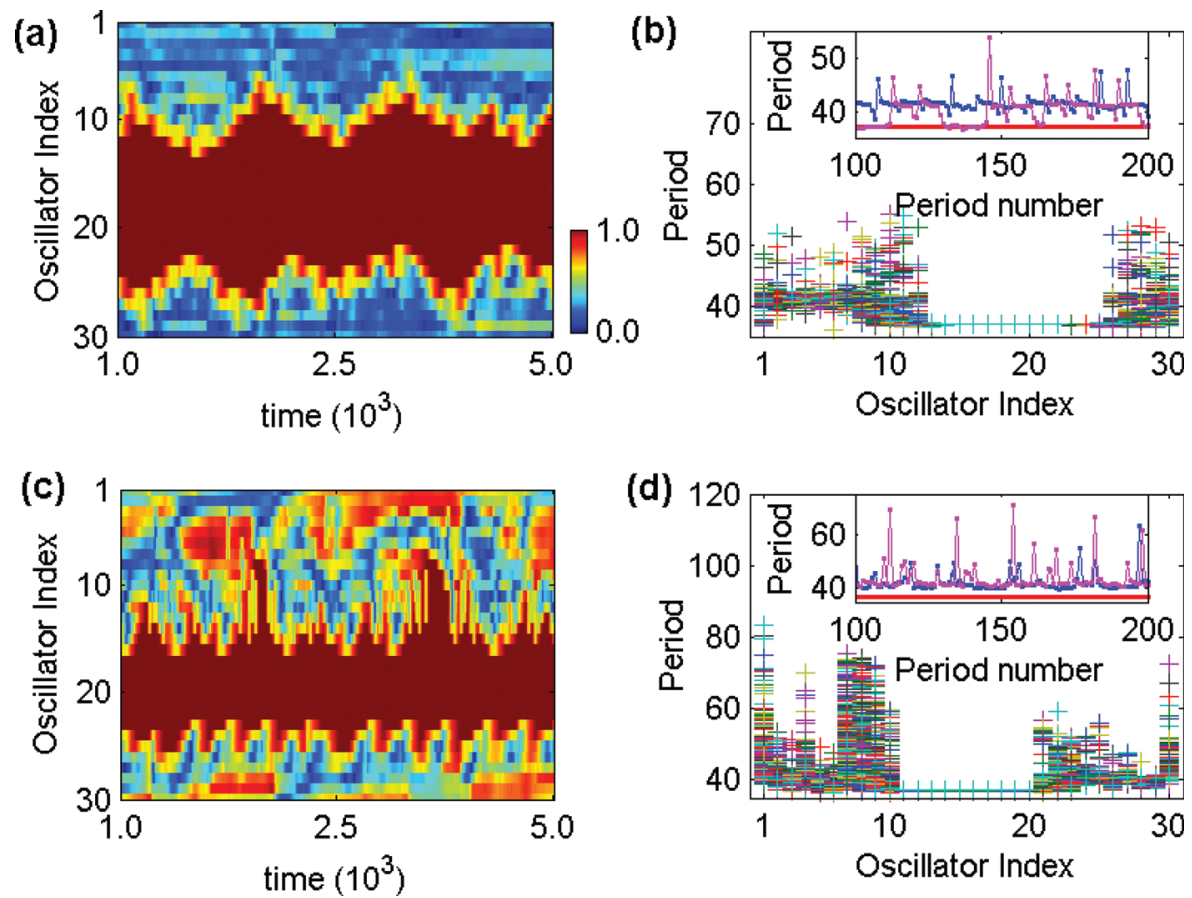

FIG. 2. Simulations showing simple chimera behavior with 30 homogeneous and 30 heterogeneous BZ oscillators, with a period of 41.0 and a mean period and standard deviation of $41.0 \pm 2.1$, respectively. Each simulation was started with a random initial phase distribution of the oscillators. (a) Local order parameter as a function of time for homogeneous oscillators. (b) Scatter plot of 500 consecutive periods for each oscillator in (a); inset shows the length of consecutive periods of a synchronized oscillator $(j=20$, red) and two asynchronous oscillators $(j=5$, magenta; $j=30$, blue). (c) Local order parameter as a function of time for heterogeneous oscillators. (d) Scatter plot of 500 consecutive periods for each oscillator in (c); inset shows the length of consecutive periods of a synchronized oscillator $(j=20$, red) and two asynchronous oscillators $(j=5$, magenta; $j=30$, blue). Note that the oscillator index is shifted by 5 oscillators from (c) to (d). Numerical parameters: $n=10, \kappa=0.4, \tau=35.0, K^{\prime}=6.3 \times 10^{-5}$, and $\phi_{0}=5.3 \times 10^{-4}$.

(a)

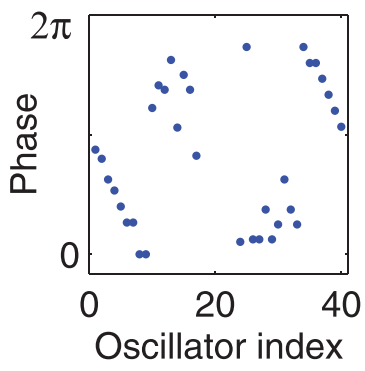

(d)

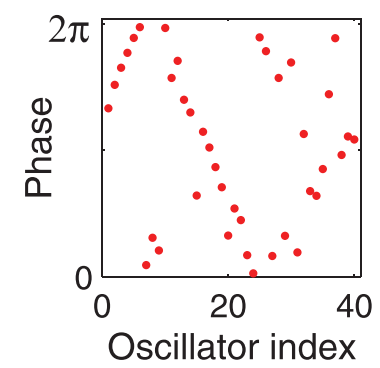

(b)

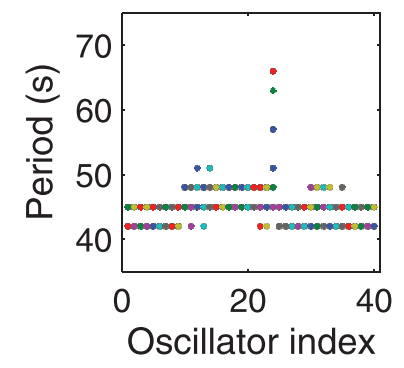

(c)

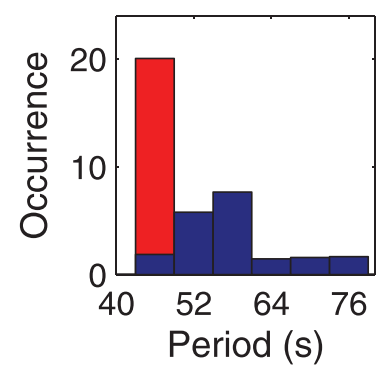

(e)

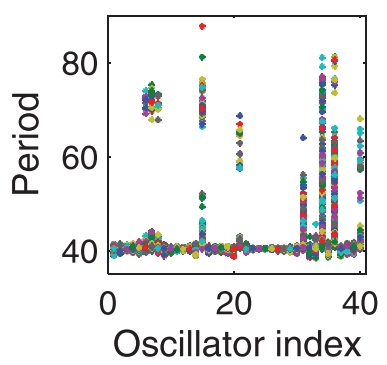

(f)

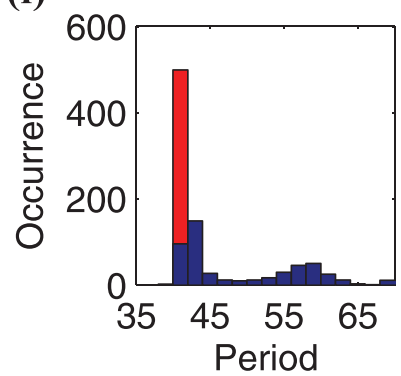

FIG. 3. Experimental measurements illustrating chimera-like states with phase waves in a system of 40 coupled BZ oscillators. (a) Snapshot of the phase of each oscillator at $t=1200 \mathrm{~s}$. (b) Period scatter plot over 20 periods. (c) The distribution of periods for 20 consecutive periods for an oscillator in the phasewave group $(j=35$, red) and for an oscillator in the incoherent group $(j=24$, blue). Simulations illustrating chimera-like states with phase waves in 40 coupled heterogeneous BZ oscillators. (d) Phase snapshot showing coexisting phase waves at $t=9.0 \times 10^{4}$. (e) Period scatter plot over 500 consecutive periods. This plot reveals the presence of several unsynchronized oscillators with a wide period distribution within the phase-wave region. (f) Period distribution for an oscillator in a phase-wave region $(j=27$, red) and in an incoherent region $(j=36$, blue). Experiment parameters: $\kappa=0.6$. Simulation parameters: $\kappa=0.4$. Other parameters as in Figs. 1 and 2. 
This state is composed of a phase-wave region of frequency synchronized oscillators, with an approximately constant phase difference between neighboring oscillators, coexisting with a region of unsynchronized oscillators. The phase snapshot in Fig. 3(a) shows oscillators indexed $j=34-48$ $(\bmod 40)$ to be in a phase-wave structure, while oscillators indexed $j=9-33$ are asynchronous. The period scatter plot in Fig. 3(b) shows that the period of the oscillators in the phase wave is shorter, on average, than the period of the unsynchronized oscillators. Figure 3(c) shows a histogram of the period distributions of two of the oscillators, one in the phase-wave region and one in the incoherent region. The phase-wave oscillator has a shorter mean period and a narrower period distribution compared with the incoherent oscillator.

Chimera-like states with phase waves are also seen in simulations with heterogeneous oscillators. Figures 3(d)-3(f) show the corresponding behavior from a simulation in which several phase-wave structures can be seen. Oscillators embedded within the phase wave again have a shorter mean period and a narrower period distribution than the unsynchronized oscillators, as shown in Fig. 3(f), much like in the experimental system. The phase and period plots in Figures 3(d) and 3(e) show that the regions of incoherent oscillators and phase-wave oscillators are less well defined than in simple chimera states. However, the region of frequencysynchronized, phase-ordered oscillators coexisting with a region of aperiodic oscillators is quite similar to a simple chimera state, with frequency-synchronized, phase-synchronized oscillators coexisting with a region of aperiodic oscillators.

We note that phase waves differ from splay states ${ }^{41-45}$ in that only a fraction of the oscillators are phase-wave synchronized, while the remaining oscillators are unsynchronized. We have not observed splay states in simulations with heterogeneous oscillators or in our experiments; however, with special initial conditions, we have observed stable splay states in the homogeneous system. Simulations with 40 oscillators exhibited splay states that were stable to small perturbations, where the initial conditions were similar to the final stable state.

\section{PHASE-CLUSTER CHIMERAS AND PHASE-SLIP BEHAVIOR}

Multiple phase-cluster chimera states, also known as multi-chimera states, are composed of more than one phase synchronized group of oscillators separated by incoherent oscillators. ${ }^{16,18,37}$ The oscillators separating the synchronized groups interact with the clusters and their immediate neighbors. Figure 4(a) shows a 2-phase cluster chimera state from a simulation with homogeneous oscillators, with the incoherent oscillators indexed $j=14-17$ and $j=32-37$. The size of the clusters fluctuates with time, as the synchronized oscillators recruit neighboring unsynchronized oscillators or lose terminal synchronized oscillators. The incoherent oscillators have a much larger spread in period and a longer mean period than the synchronized oscillators, as shown in Fig. 4(b). Figure 4(c) shows the irregular variations in the period of oscillator $j=33$ with time. Phase-cluster and multi-headed chimeras are also seen in the heterogeneous model system and in experiments. ${ }^{27,37}$ Figures 4(d) and 4(e) show, respectively, period scatter plots for 2-headed and 3-headed chimeras from simulations and an experiment.

Phase-slip behavior is characterized by oscillators periodically slipping from phase clusters. ${ }^{46}$ Figure 5(a) shows a snapshot of phase-slip behavior, oscillators $j=15,16,35$, and 36, in a system of homogeneous oscillators. This behavior is strikingly similar to the phase-cluster chimera behavior
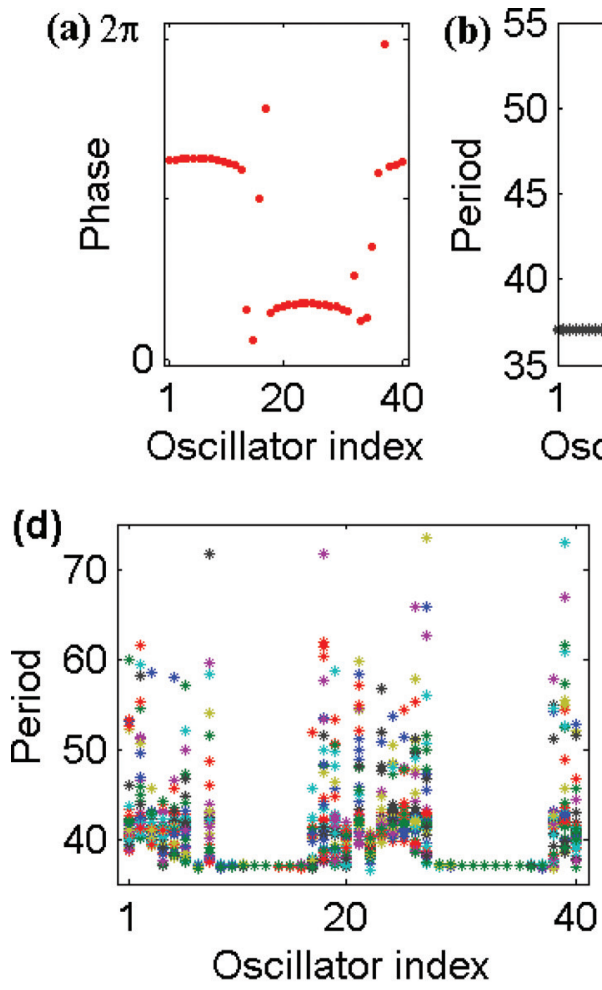
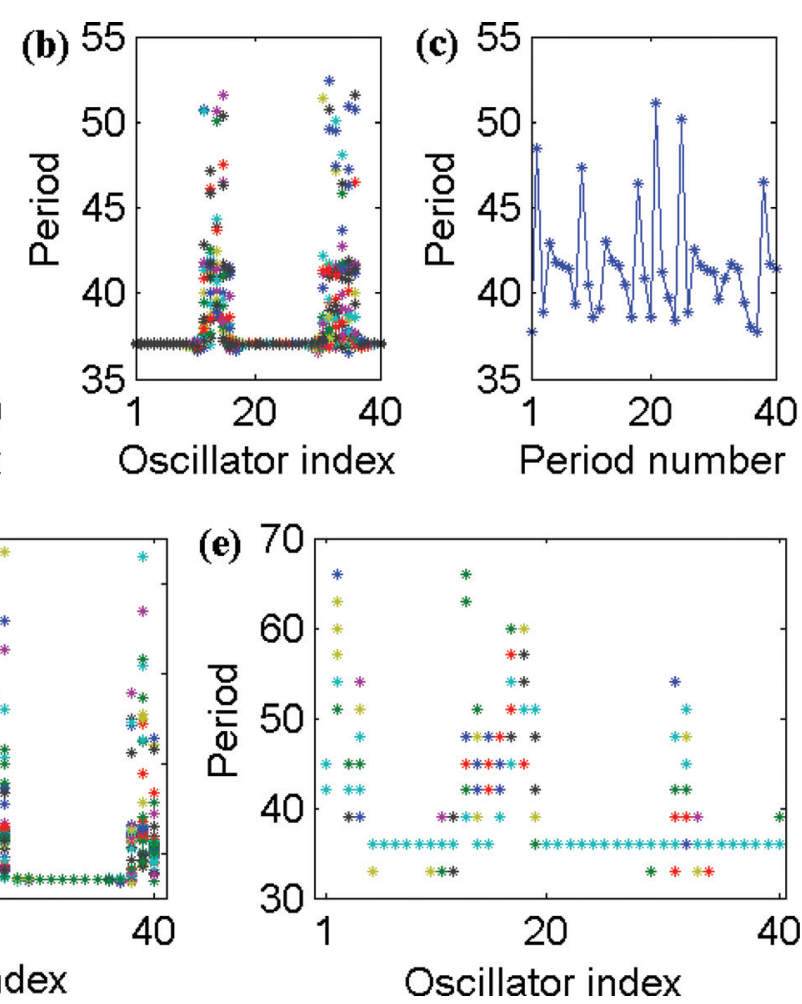

FIG. 4. Phase-cluster chimera states in systems of homogeneous and heterogeneous oscillators. (a) Snapshot showing the phase of each oscillator at $t=1.80$ $\times 10^{4}$ in a system of 40 homogeneous oscillators. (b) Scatter plot of oscillator periods for 1000 consecutive cycles. (c) Period as a function of period number for oscillator $j=33$. (d) Period scatter plot taken over 500 cycles from simulations with 40 heterogeneous oscillators. Both simulations were started with a random initial phase distribution. (e) Experimentally observed phase-cluster chimera state. Period scatter plot taken over 20 cycles in a system of $40 \mathrm{BZ}$ oscillators. Simulation parameters: $\kappa=0.4$. Experiment parameters: $\kappa=0.8$. Other parameters and conditions as in Figs. 1 and 2. 
(a) $2 \pi$

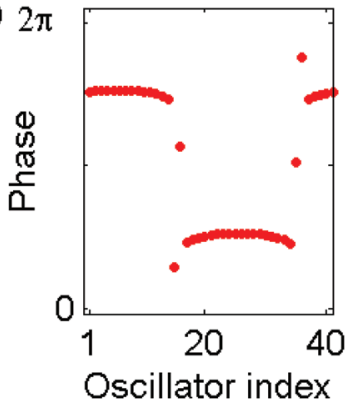

(d) $2 \pi$

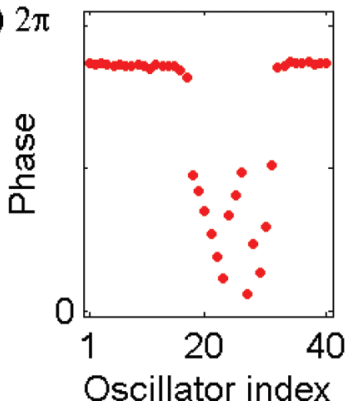

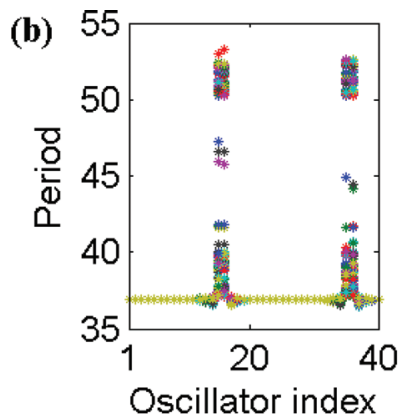

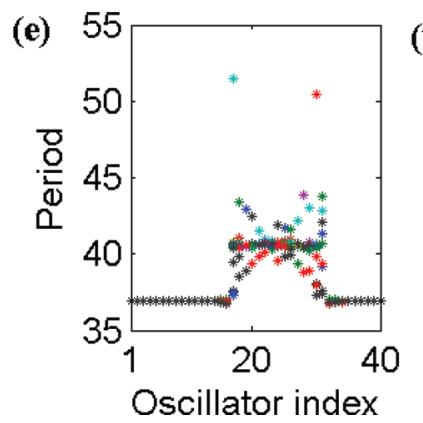

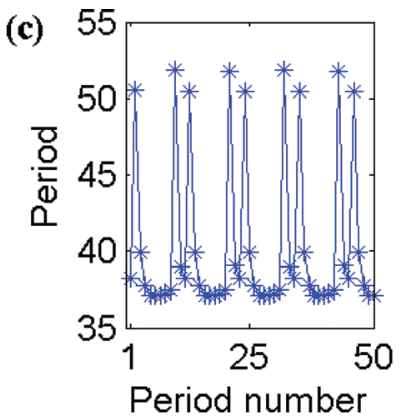

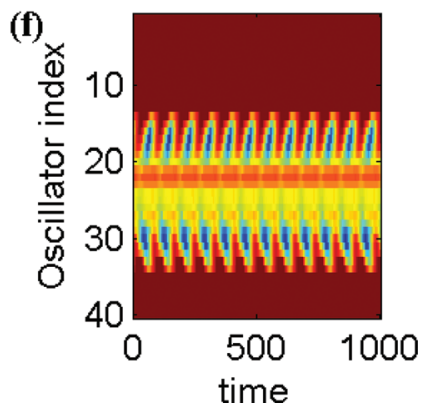

FIG. 5. Phase-slip behavior in systems of homogeneous and heterogeneous oscillators. (a) Snapshot showing the phase of each oscillator at $t=1.7 \times 10^{4}$ in a system of 40 homogeneous oscillators. (b) Period scatter plot over 500 consecutive periods. (c) Period as a function of period number for oscillator $j=15$. Simulation parameters: $\kappa=0.35$. Other parameters as in Fig. 2. (d) Phase snapshot taken at $t=4.5 \times 10^{5}$ in a system of 40 heterogeneous oscillators. (e) Period scatter plot over 500 consecutive periods. (f) Local order parameter over 133 periods revealing higher order periodicity of the oscillators adjacent to the synchronized region. Simulation parameters: $\kappa=0.4$. Other parameters as in Fig. 2. in Fig. 4(a). As the oscillators slip between the clusters, they have intermittent encounters with the clusters, causing large variations in period, as shown in Fig. 5(b). The phaseslip behavior leads to the oscillators having a longer average period than the oscillators within the clusters. A plot of the period of an individual slip oscillator in Fig. 5(c) shows that the oscillator exhibits period-11 oscillations. While the phase-slip behavior appears to be similar to a phasecluster chimera state, ${ }^{1,2}$ analysis of individual oscillators reveals the coexistence of two groups of synchronized oscillators with simple and complex periodicity. We note that $\kappa=0.40$ for the phase-cluster chimera state in Figs. 4(a)-4(c), while $\kappa=0.35$ for the phase-slip behavior in Figs. $5(a)-5(c)$. The lower value of $\kappa$ leads to a larger range of coupling and greater synchronization, resulting in fewer oscillators between the phase clusters, which become phase locked with complex periodicity.

Phase-slip states are also seen in model simulations with heterogeneous oscillators. An example of phase-slip behavior is shown in Figs. 5(d)-5(f), with periodic switching between an in-phase cluster and a region of out-of-phase oscillators. In Fig. 5(d), oscillators $j=1-17$ and $j=32-40$ form the synchronized group, while oscillators $j=21-27$ are approximately phase locked. The oscillators in this phaselocked region have a higher mean period than the synchronized oscillators. Oscillators $j=18-20$ and $j=28-31$ show a broader distribution of periods, corresponding to complex periodicity behavior, as they move under the influence of the phase-locked and synchronized groups (Fig. 5(e)). The periodicity of this behavior is revealed by the spatial order parameter plotted over multiple cycles, as shown in Fig. 5(f).

Characterization of phase-slip behavior in the experimental system is problematic due to experimental noise, making the distinction between complex periodicity and aperiodicity difficult. A 40 oscillator system was used in our experiments, and the simulations with homogeneous and heterogeneous oscillators in Fig. 5 also used 40 oscillator systems, suggesting that size effects are not as important as the inherent experimental noise. It is possible that the inherent experimental noise causes complex-periodicity slip behavior to appear aperiodic in the experiments.

\section{MIXED STATES}

Regions of incoherent, synchronized, and phase-wave behavior may interact to produce very complex behavior. The probability of such mixed states generally increases as the number of oscillators increases. In addition, in larger populations of oscillators, an initial random phase distribution may evolve to reveal two categories of seemingly unsynchronized oscillators, one that is aperiodic and another that is simply phase dispersed. An example of this is shown in Figs. 6(a)-6(c) in a simulation of 90 homogeneous oscillators. In contrast to the smaller homogeneous system shown in Fig. 2, this system exhibits two distinctly different types of behavior outside the synchronized region. Groups of asynchronous oscillators, $j=51-61$ and $j=76-83$, which have a broad distribution in period, are seen immediately adjacent to the synchronized region. This asynchronous behavior is similar to that in Fig. 2; however, outside of this region, $j=1-51$ and $j=84-90$, the oscillators are simply phase dispersed and oscillate close to their natural period. Figure 6(c) shows the period as a function of period number of 4 oscillators at different locations. The oscillator fartherest from the synchronized phase cluster $(j=40$, green) oscillates with its natural period. Hence, although it is phase dispersed, it is relatively unaffected by the synchronized oscillators in the phase cluster or by its phase dispersed neighbors. Aperiodic oscillations are exhibited by oscillators at the boundary of the phase cluster, where the oscillations switch aperiodically between approximately the natural period and the period of the synchronized phase cluster. The oscillator farther from the phase cluster $(\mathrm{j}=58$, blue) spends more time oscillating approximately at its natural period (green), while the oscillator closer to the phase cluster $(j=60$, pink) spends more time 
(a)

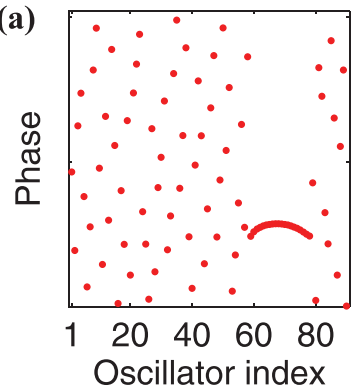

(d)

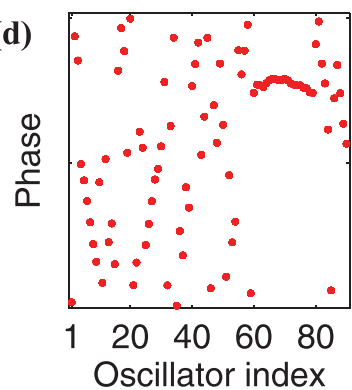

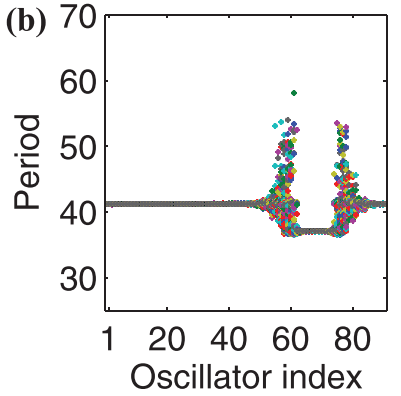
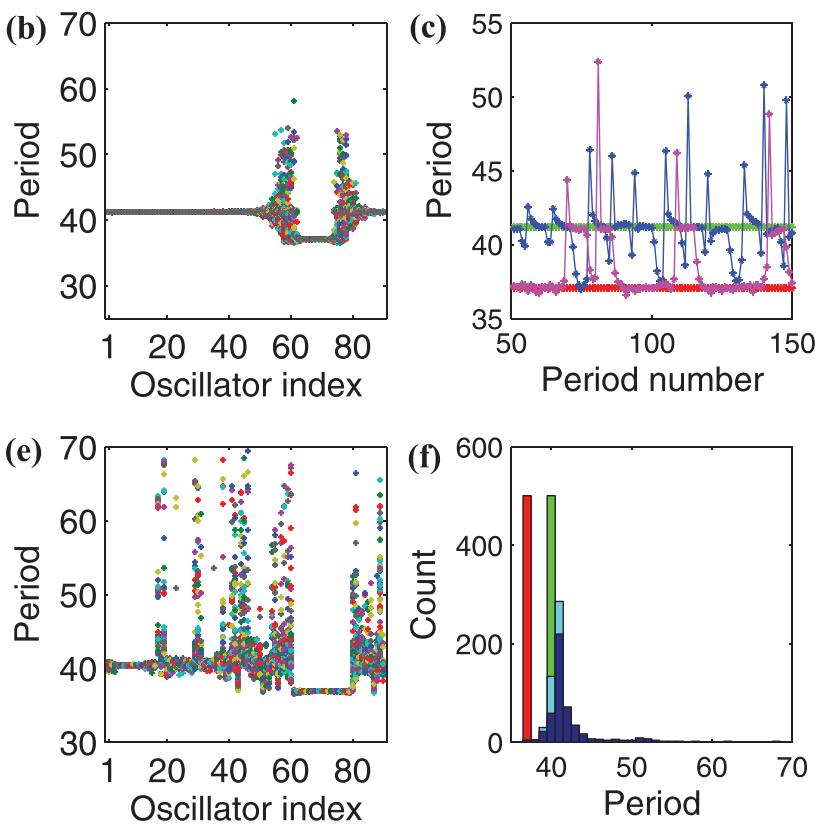

FIG. 6. Simulations of chimera behavior in larger systems of homogeneous and heterogeneous oscillators. (a) Snapshot of the phase of each oscillator at time $2.9 \times 10^{5}$ in a simulation with 90 homogeneous oscillators. (b) The corresponding scatter plot for 500 periods of each oscillator. (c) Period as a function of period number for 100 consecutive periods of 4 oscillators: $j=40$ (green) oscillates approximately with its natural period, $j=58$ (blue) and $j=60$ (pink) exhibit aperiodic behavior, switching between the natural and synchronized periods, and $j=66$ (red) oscillates with the synchronized phase cluster period. (d) Snapshot of the phase of each oscillator at time $2.9 \times 10^{5}$ in a simulation with 90 heterogeneous oscillators. (e) The corresponding scatter plot for 500 periods of each oscillator. (f) Period histogram for four oscillators showing following behaviors: synchronized ( $j=75$, red), phase wave $(j=10$, green), asynchronous $(j=58$, dark blue), and phase dispersed and periodic $(j=50$, light blue). Numerical parameters, $\kappa=0.4$, and other parameters as in Fig. 2.

oscillating approximately with the period of the synchronized phase cluster (red). Of course, an oscillator within the phase cluster $(j=66$, red) oscillates with the synchronized period. Hence, the aperiodicity arises from an interplay between the synchronized oscillators at the boundaries of the phase cluster and the phase dispersed oscillators, with the predominate period shifting from the synchronized period (red) to the natural period (green) with increasing distance from the phase cluster boundary.

We note that while the 30 oscillator system in Figs. 2(a) and 2(b) exhibits only periodic synchronized oscillators and aperiodic unsynchronized oscillators, as seen in typical examples of simple chimera states, a 40 oscillator system is large enough to also exhibit some phase dispersed oscillators that oscillate at approximately their natural period. The range of the aperiodic oscillators at the boundaries of the synchronized phase cluster is approximately equal to the coupling radius, which is largely determined by the value of $\kappa$ in Eq. (1). The system in Figs. 2(a) and 2(b) is small enough that the range of aperiodic oscillators extends from one boundary of the synchronized phase cluster to the other boundary.

An example of mixed dynamical behavior in a group of 90 heterogeneous oscillators is shown in Figs. 6(d)-6(f). The phase snapshot shown in Fig. 6(d) reveals a synchronized group and regions of phase-wave activity, together with regions of aperiodicity. A plot of the period of each oscillator is shown in Fig. 6(e), where a number of regions with narrow period distributions are separated by regions of asynchronous oscillators with broad period distributions. Oscillators $j=1-18$ and $j=22-28$ have narrow period distributions with mean periods less than the average natural period of the uncoupled oscillators but greater than that of the synchronized group. Examination of Fig. 6(d) shows that oscillators $j=1-11$ and $j=22-28$ form a pair of phase waves with opposite slopes. The remaining oscillators, $j=12-18$, are effectively phase-locked to the phase waves. Immediately adjacent to these regions of phase locked behavior are asynchronous regions with broad period distributions.

A synchronized region occurs for oscillators $j=61-80$. Figure 6(f) illustrates that the period of the synchronized oscillators is smaller than that of an oscillator in the phasewave region. In contrast to the homogeneous system, no clear trend is seen in the distribution of period as a function of distance from the synchronized group. Example oscillators are shown in Fig. 6(f), with one in the synchronized group (red), one in a phase wave (green), an asynchronous oscillator (blue) immediately adjacent to the synchronized group, which shows a broad period distribution with no bias toward the period of the synchronized group, and a phase dispersed oscillator (light blue) with a mean period close to its natural period.

Mixed behavior is typically not seen in simulations with smaller numbers of oscillators. Similarly, in the experimental system $(N=40)$ we have not observed long lived mixed states. Transient combinations of synchronized, phase wave, and asynchronous behavior have been observed but typically last for only about 15 periods. This is likely the result of the smaller number of oscillators and experimental noise.

\section{TRAVELING PHASE-CLUSTER CHIMERAS}

In simulations of a system of 40 homogeneous oscillators, the chimera state with one phase cluster of 


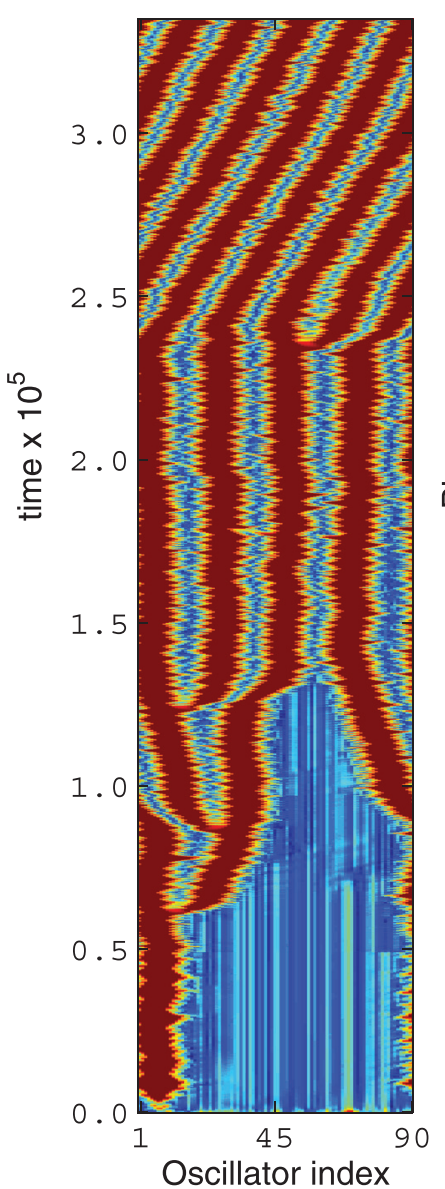

(a) (iv)

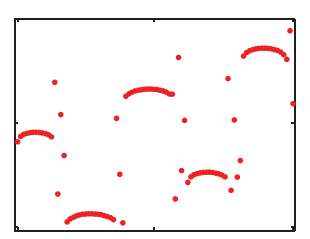

(iii)

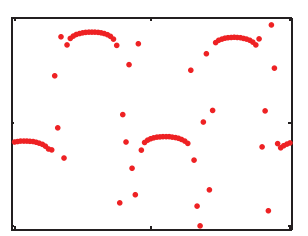

(ii)

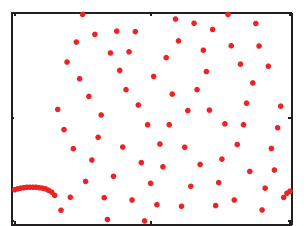

(i)

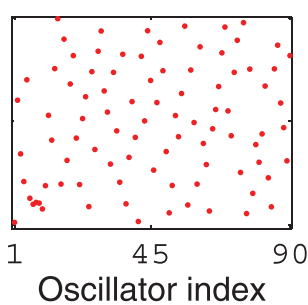

(b)

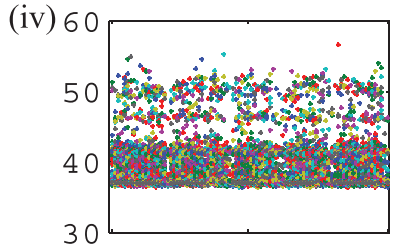

(iii)

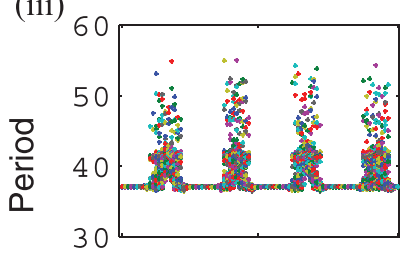

(ii)

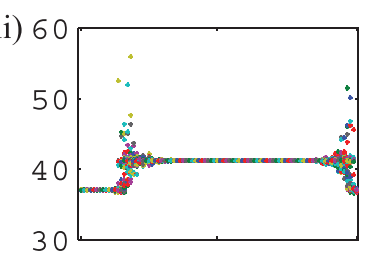

(i)

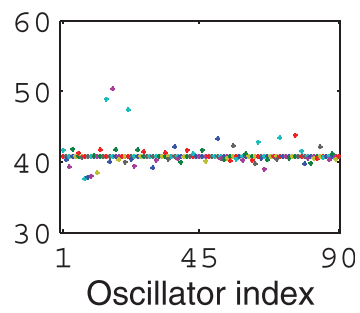

(c)
FIG. 7. Simulations with 90 coupled identical model $\mathrm{BZ}$ oscillators in a ring configuration. (a) Local order parameter $\mathrm{R}$ as a function of time. (b) Snapshot of the phase of each oscillator at four different times: $t=3.0 \times 10^{3}, 4.0 \times 10^{4}$, $2.0 \times 10^{5}$, and $3.2 \times 10^{5}$. (c) Scatter plot of each oscillator for 500 periods at four different time intervals that are near the times in (b). Simulations are initiated with a random initial phase distribution and are carried out for $\sim 10^{4}$ oscillation periods. Variations in other system parameters such as $\kappa$ can lead to traveling phase-cluster states with different numbers of clusters and oscillator occupancies. Simulation parameters $\kappa=0.4$, with other parameters as in Fig. 2.

synchronized oscillators together with primarily asynchronous oscillators is typically found to be stable for the duration of the simulation. However, in a small number of cases, the 1-phase cluster chimera is found to undergo a transition, where the existing cluster region develops into two new phase-cluster regions that are antiphase. In the cases where a 2-phase cluster chimera state is formed, the state is typically stable for the duration of the simulation. However, occasionally another transition occurs in which three phasecluster regions develop, which are $2 \pi / 3$ out of phase. In the cases where a 3-phase cluster chimera state forms, the entire pattern rotates around the ring of oscillators with an approximately constant angular velocity. This traveling phase-cluster chimera, once formed, persists for the duration of the simulation. Similar phenomena are observed as $N$ is increased, with the number of transitions to higher cluster states increasing and the traveling phase clusters occurring with higher n-cluster states.

The transitions to increasingly higher n-cluster states and a traveling 5-phase cluster chimera are illustrated in simulations of a ring of 90 homogeneous oscillators, shown in Fig. 7. A cluster of synchronized oscillators spontaneously forms at $t=300$, as shown in Fig. 7(a). The phase cluster is made up of $13 \pm 2$ oscillators and exhibits small size fluctuations. A second cluster spontaneously grows from the first to give a 2-phase cluster state at $\sim 0.6 \times 10^{5}$. A third cluster then grows from the recently formed cluster to give a 3-phase cluster state at $\sim 0.8 \times 10^{5}$. A 4-cluster state is formed from

another spontaneous growth event at $\sim 1.2 \times 10^{5}$, which is more persistent than the earlier states. It is a 2-headed, 2-phase cluster chimera with pairs of antiphase clusters. We have been unable to discern any predictable timing pattern in new cluster formation, either within or between simulations.

The formation of the 5-cluster state at $\sim 2.4 \times 10^{5}$ initiates a drifting motion, with the pattern rotating counterclockwise around the 90-oscillator ring. Figure 7(b) shows snapshots of the phase of each oscillator at four different times corresponding to some of the states in Fig. 7(a). Figure 7(c) shows scatter plots of 500 periods of each oscillator, with the states corresponding to the snapshots in Fig. 7(b). As can be seen in Fig. 7(c-i), the oscillators each have approximately the same period before the first phase cluster is spontaneously formed. The synchronized oscillators within a phase cluster exhibit an average period that is shorter than the average natural period, as shown in Fig. 7(c-ii). Also shown in this figure are the regions of aperiodic oscillators, which occur at each end of the phase cluster.

The 4-cluster state, shown in Fig. 7(c-iii), reveals the same period for the synchronized oscillators within the clusters and a broad period distribution for the asynchronous oscillators between the clusters. Formation of the fifth cluster initiates the counter-clockwise motion of the 5-phase cluster pattern, with phase differences of $2 \pi / 5$ between clusters. In the traveling phase-cluster pattern, each individual oscillator exhibits intervals of periodic and intervals of aperiodic behavior, as the clusters move through the oscillators. 
Hence, each oscillator has approximately the same period distribution, as shown in Fig. 7(c-iv).

The transitions giving rise to additional phase clusters are observed in populations of 40 to 90 homogeneous oscillators in our simulations, with the maximum number of clusters increasing with increasing $N$. For $N=40$, the maximum number of clusters is three, whereas for $N=90$, the maximum number is five, for the parameters in Fig. 7. In these cases, the traveling phase clusters are observed when the maximum number of clusters is attained. The linear velocity of the rotation, defined as the number of oscillators divided by the time required for one complete rotation of the pattern around the oscillator ring, is found to be a decreasing function of $N$.

We have found no evidence of traveling clusters in a 4-cluster chimera state, which corresponds to the maximum number of clusters observed with $70 \leq N<90$. We also note that completion of all the transitions to the maximum number of phase clusters does not occur in most simulations. For example, in a set of 200 simulations with 40 homogeneous oscillators, using special initial conditions with a group of synchronized oscillators, $29 \%$ of the simulations resulted in a single transition to a 2-phase cluster chimera state. A second transition to a 3-phase cluster chimera state was observed in $10.5 \%$ of the simulations. Similar rotating patterns have been observed in studies of nonlocally coupled complex Ginzburg-Landau oscillators, ${ }^{6}$ although no equivalent transition sequences were reported. Traveling phasecluster chimera states have not been observed in our experiments and simulations with heterogeneous oscillators.

\section{DISCUSSION}

The occurrence of chimera and chimera-like states described in this paper is dependent on several key factors, including the coupling decay constant $\kappa$, the system size, and the initial conditions. Figure 8 shows behavior observed with different values of $\kappa$ for a 40-oscillator ring system with random initial phase distributions (except for splay states and phase waves). The value of $\kappa$ controls the range of nonlocal coupling in our system, with smaller values corresponding to stronger interactions over a greater range of nonlocal neighbors. The various types of chimera and chimera-like states generally occur for $0.3 \leq \kappa \leq 0.6$ in simulations of homogeneous and heterogeneous systems, while the range is somewhat larger in the experiments. For larger values of $\kappa$, there is insufficient nonlocal coupling to support the behavior, and simple phase dispersed behavior dominates. For smaller values of $\kappa$, the greater coupling range gives rise to the fully synchronized state.

Multistability occurs across a range of $\kappa$ values, with the fully synchronized state coexisting with other states, particularly at lower values of $\kappa$. For example, at $\kappa=0.4$, the homogeneous oscillator system exhibits the following states: fully synchronized, chimera, phase-cluster chimera, traveling phase-cluster, phase-slip, and splay state behavior. The heterogeneous oscillator system at this value of $\kappa$ exhibits these states and also the chimera-like state with phase waves but does not exhibit the traveling phase-cluster

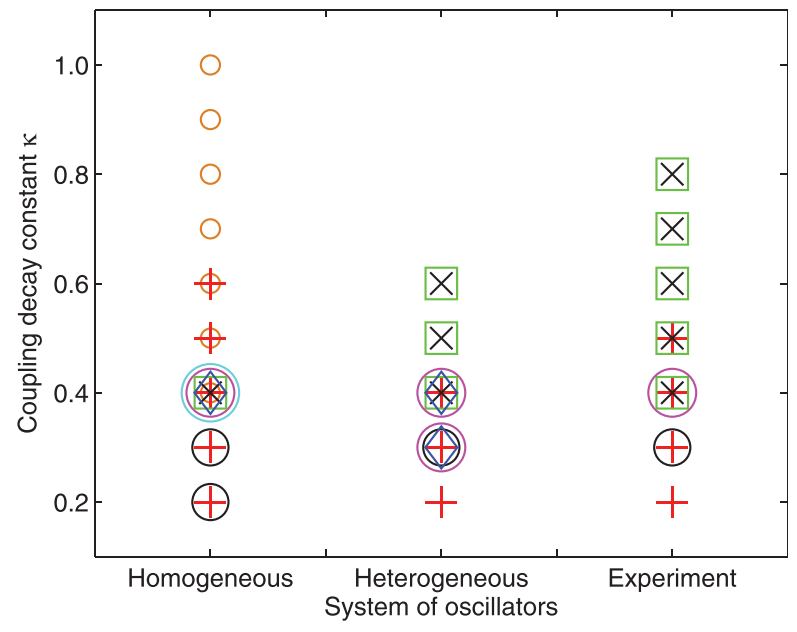

FIG. 8. Summary of the dynamical states observed in simulations of the homogeneous and heterogeneous oscillator systems and the experimental system for different values of $\kappa$, indicated by the following symbols: fully synchronized (red + ), synchronization clusters (black $\bigcirc$ ), splay state (orange $\bigcirc$ ), chimera (black $\times$ ), phase-cluster chimera (green $\square$ ), traveling phase-cluster chimera (blue $\bigcirc$ ), chimera-like states with phase waves (magenta $\bigcirc$ ), phase slip behavior, (blue $\diamond$ ). Multistability is exhibited at many values of $\kappa$. Simulations and experiments for all of the states except splay states and phase-wave states (homogeneous system) were started from random initial phase distributions. For splay states and phase-wave states, simulations were started from a splay-state-like initial phase distribution. Other experimental parameters are as in Fig. 1; other simulation parameters are as in Fig. 2.

or splay states. The experimental system at $\kappa=0.4$ exhibits the same states as the heterogeneous model system; however, it has not been possible to identify phase-slip behavior.

Special initial conditions can increase the probability of finding a chimera state. For example, in the homogeneous system, the probability of finding a chimera state increases significantly with initial conditions that reflect the targeted state, ${ }^{10,15}$ compared with a random initial phase distribution. Increasing $N$ leads to only a small increase in the probability of finding a chimera state. In contrast, with heterogeneous oscillators, using special initial conditions does not significantly influence the probability of finding a chimera state, while increasing $N$ increases the probability.

Our observations of the different experimental chimera and chimera-like states are mirrored by the behavior observed in the simulations of the heterogeneous and homogeneous oscillator systems, although several significant differences exist. For example, splay states and traveling phase-cluster chimeras have not been observed in the heterogeneous system or in the experiments. Also, the extent of the periodic phase-dispersed region that coexists with coherent and incoherent regions is smaller in both the experimental system and simulations of the heterogeneous system compared with the homogeneous system.

Each of the states observed in the experimental system, once formed, normally lasts for the duration of the experiment, which is typically about $30 \mathrm{~min}$. Simulations are generally run for 2500 periods and, similarly, once formed, states last for the duration of the simulation. Long term stability was tested by running simulations for 12500 periods for each of the observed states in Fig. 8 for $\kappa=0.4$. With 
the exception of the chimera-like states with phase waves and the phase-cluster chimeras in the system of heterogeneous oscillators, each state, once formed, lasted for the duration of simulation. The chimera-like states with phase waves, such as that shown in Fig. 3(d), lasted for about 2500 oscillations, and then collapsed to a fully synchronized state in the longer simulation. However, phase wave behavior formed as part of a mixed state, such as that shown in Fig. $6(d)$, lasted for the duration of the longer simulation. The existence of the traveling phase-cluster states and the associated transitions indicates that the phase-cluster chimera states also may not be long-term stable. Other studies have suggested that certain chimera states are long transients, with the length of the transient increasing with the size of the system. $^{13,17,27}$

In all of the states observed, the asynchronous behavior typically associated with a chimera is found to be localized, i.e., within the coupling radius of the ordered region. A phasedispersed state tends to occur beyond this region of influence, although the distinction between the different apparently aperiodic regions is not immediately obvious from the phase snapshot. An analysis of the period or frequency distribution is necessary to distinguish between these behaviors as well as between the phase-cluster chimera and the complex-periodicity, phase-slip behavior. The simple chimera state is but one of many chimera and chimera-like states in systems of non-locally coupled chemical oscillators.

\section{ACKNOWLEDGMENTS}

This material is based on work supported by the National Science Foundation (Grant No. CHE-1565665).

\section{APPENDIX A: EXPERIMENTAL AND COMPUTATIONAL PROCEDURES}

\section{Instrumentation}

The experimental set-up consists of a modified video projector (SLM) with a 440-460 nm band pass filter, beam splitter, camera (CCD), computer (PC), and the reactor. The camera and the modified projector are controlled via the computer. The camera is placed above the beam splitter and a $2 \mathrm{~cm}$ diameter reaction vessel to record images of the state of each oscillator in real time. The set-up is shown in Fig. 9.

a)

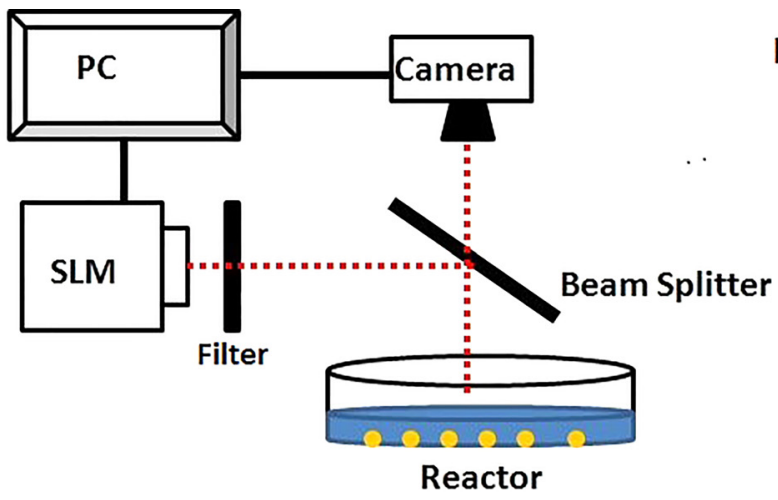

The CCD camera is connected to a PC, which processes the images and computes the feedback illumination for each particle. The feedback illumination is applied through a spatial light modulator (SLM). The light from the SLM passes through the band-pass filter to a beam splitter that reflects the light onto the particles in the reactor.

\section{Preparation of ruthenium(II) catalyst-loaded beads}

The catalyst solution of $25.0 \mathrm{mM} \mathrm{Ru}(\mathrm{bpy})_{3}^{2+}$ is prepared by dissolving $0.47 \mathrm{~g}$ of tris(2-2'-bipyridyl)ruthenium(II) chloride hexahydrate ( $98 \%$ complex) in $25.0 \mathrm{ml}$ of doubly distilled water. DOWEX 50WX2-100 ion exchange beads $(3.0 \mathrm{~g})$ are mixed with $10.0 \mathrm{ml}$ of $2.50 \mathrm{mM}$ ruthenium(II) catalyst solution $\left(8.3 \times 10^{-6} \mathrm{~mol} / \mathrm{g}\right.$ resin $)$. The mixture is stirred at a low speed for $24 \mathrm{~h}$ and then filtered. The catalyst-loaded particles are washed with distilled water before drying at room temperature for $24 \mathrm{~h}$.

\section{Experimental procedures}

Stock solutions of $1.0 \mathrm{M}$ sodium bromide $(\mathrm{NaBr}), 1.5 \mathrm{M}$ sodium bromate $\left(\mathrm{NaBrO}_{3}\right)$, and $3.0 \mathrm{M}$ sulfuric acid $\left(\mathrm{H}_{2} \mathrm{SO}_{4}\right)$ are prepared with reagent-grade chemicals (Fischer Scientific) and doubly distilled water. The solutions are stored at room temperature. Malonic acid (MA) is prepared just before beginning an experiment because it is not stable for periods greater than approximately $24 \mathrm{~h}$. The catalyst-free BZ solution is prepared with $0.77 \mathrm{M} \mathrm{H}_{2} \mathrm{SO}_{4}, 0.51 \mathrm{M} \mathrm{NaBrO}_{3}, 0.08 \mathrm{M} \mathrm{NaBr}$, and $0.16 \mathrm{M}$ MA.

The catalyst-loaded particles are randomly distributed in a BZ catalyst-free solution. The particles are positioned at more than three diameters apart to eliminate local diffusive coupling. The particles are in an oscillatory state under the experimental conditions. Experiments are carried out with 40 particles, which oscillate independently before they are coupled. The resulting initial conditions are close to a random initial phase distribution, although not mathematically random as in our model simulations. For special initial conditions with synchronized oscillators, a group of oscillators is synchronized by strongly coupling them with a low value of $\kappa$ before introducing the regular coupling scheme. A computer algorithm is used to align the camera and projector coordinate systems to allow real-time feedback by illumination of individual oscillators. The particles are mapped onto a virtual array, which is used to set up a desired network with a defined coupling.

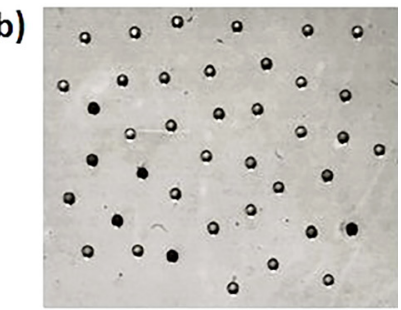

FIG. 9. Experimental set-up. The camera (CCD), connected to the computer $(\mathrm{PC})$, records the position and intensity of the catalyst-loaded beads in the reactor. The image is processed by the PC and feedback is applied via the spatial light modulator (SLM) through the filter. (b) Catalyst-loaded particles recorded by the CCD camera. 
In the experiments, images are captured every $3.0 \mathrm{~s}$ with the projected feedback briefly interrupted to allow image capture. A background light intensity of $1.4 \mathrm{~mW} \mathrm{~cm}^{-2}$ is used for capturing images. The variation in transmitted light intensity by individual particles is monitored as a function of time. The observed oscillations in light intensity are the result of ruthenium(II) (orange) being oxidized to ruthenium(III) (green), which is then reduced back to ruthenium(II). The molar extinction coefficient for ruthenium(II) is higher than that for ruthenium(III), and the maximum in transmitted light intensity therefore corresponds to the maximum in ruthenium(III) concentration. The captured image is analyzed to determine the intensities, in gray level, associated with each oscillator. The intensities are used to compute the feedback based on the coupling scheme. The computed illumination is sent to the projector, which illuminates each particle independently according to

$$
\phi_{j}=\phi_{0}+\phi_{f b, j}
$$

where $\phi_{j}$ is the projected light intensity, $\phi_{0}$ is the background light intensity, and $\phi_{f b, j}$ is the computed feedback for oscillator $j .{ }^{27,36,37}$

\section{Simulation model and procedures}

Simulations were carried out with the two-variable ZBKE model ${ }^{39}$ modified to describe the discrete photosensitive BZ oscillator system, ${ }^{27,36}$ where

$$
\begin{aligned}
\epsilon_{1} \frac{d X_{j}}{d t}= & \phi_{j}-X_{j}^{2}-X_{j}+\epsilon_{2} \gamma u_{s s}^{2}+u_{s s}\left(1-Z_{j}\right) \\
& +\frac{\left(\mu-X_{j}\right)}{\left(\mu-Z_{j}\right)}\left(\frac{q \alpha Z_{j}}{\left(\epsilon_{3}+1-Z_{j}\right)}+\beta\right), \\
\frac{d Z_{j}}{d t} & =2 \phi_{j}+u_{s s}\left(1-Z_{j}\right)-\frac{\alpha Z_{j}}{\left(\epsilon_{3}+1-Z_{j}\right)},
\end{aligned}
$$

describe the chemistry of oscillator $j$. The variables $X_{j}$ and $Z_{j}$ and the constant $q_{j}$ represent $\left[\mathrm{HBrO}_{2}\right],\left[\mathrm{Ru}(\mathrm{bpy})_{3}^{2+}\right]$, and the stoichiometric coefficient, respectively, associated with oscillator $j$. The photo-excitatory feedback associated with oscillator $j$ is $\phi_{j}$. Nondimensional model parameters are $\epsilon_{1}$ $=0.11, \epsilon_{2}=1.7 \times 10^{-5}, \epsilon_{3}=1.6 \times 10^{-3}, \gamma=1.2, \alpha=0.10$, $\beta=1.7 \times 10^{-5}, \mu=2.4 \times 10^{-4}$, and a distribution in $q$ that gives a period of $41.0 \pm 2.1$. The set of ordinary differential equations are solved using the Euler method with a time step of 0.001 . The experimental period distribution is approximately Gaussian, ${ }^{40}$ and a Gaussian period distribution is used in all simulations, with a standard deviation reflecting typical experimental values.

Simulations were carried out with the ZBKE model to check whether the photochemical coupling gives rise to any amplitude effects in a chimera state, such as the mixed state shown in Figs. 6(a)-6(c). The limit cycles of a synchronized oscillator, an aperiodic oscillator, and a periodic but phase dispersed oscillator were plotted for 30 periods. The three limit cycles were indistinguishable when they were overlaid. Blowups of the maximum and minimum in the two variables, $X$ and $Z$, representing the autocatalyst $\mathrm{HBrO}_{2}$ and the oxidized catalyst $\mathrm{Ru}(\mathrm{bpy})_{3}^{3+}$, respectively, were plotted to determine the variation in the maximum amplitudes. The maximum amplitudes of $X$ and $Z$ varied by less than $0.2 \%$, with the aperiodic oscillator showing the greatest variation.

${ }^{1}$ Y. Kuramoto and D. Battogtokh, Nonlinear Phenom. Complex Syst. 5, 380 (2002).

${ }^{2}$ D. M. Abrams and S. Strogatz, Phys. Rev. Lett. 93, 174102 (2004).

${ }^{3}$ E. A. Martens, Phys. Rev. E 82, 016216 (2010).

${ }^{4}$ D. Dudkowski, Y. Maistrenko, and T. Kapitaniak, Phys. Rev. E 90, 032920 (2014).

${ }^{5}$ J. Xie, H.-C. Kao, and E. Knobloch, Phys. Rev. E 91, 032918 (2015).

${ }^{6}$ G. C. Sethia, A. Sen, and G. L. Johnston, Phys. Rev. E 88, 042917 (2013).

${ }^{7}$ G. C. Sethia and A. Sen, Phys. Rev. Lett. 112, 144101 (2014).

${ }^{8}$ A. Zakharova, M. Kapeller, and E. Schöll, Phys. Rev. Lett. 112, 154101 (2014).

${ }^{9}$ N. Yao, Z. G. Huang, C. Grebogi, and Y. C. Lai, Sci. Rep. 5, 12988 (2015).

${ }^{10}$ D. M. Abrams and S. H. Strogatz, Int. J. Bifurcation Chaos 16, 21 (2006).

${ }^{11}$ O. Omel'chenko, Y. Maistrenko, and P. Tass, Phys. Rev. Lett. 100, 044105 (2008).

${ }^{12}$ D. Abrams, R. Mirollo, S. Strogatz, and D. Wiley, Phys. Rev. Lett. 101, 084103 (2008).

${ }^{13}$ M. Wolfrum and O. E. Omel'chenko, Phys. Rev. E 84, 015201 (2011).

${ }^{14}$ I. Omelchenko, Y. Maistrenko, P. Hövel, and E. Schöll, Phys. Rev. Lett. 106, 234102 (2011).

${ }^{15}$ I. Omelchenko, O. E. Omel'chenko, P. Hövel, and E. Schöll, Phys. Rev. Lett. 110, 224101 (2013).

${ }^{16} \mathrm{~J}$. Hizanidis, V. G. Kanas, A. Bezerianos, and T. Bountis, Int. J. Bifurcation Chaos 24, 1450030 (2014).

${ }^{17}$ D. P. Rosin, D. Rontani, N. D. Haynes, E. Schöll, and D. J. Gauthier, Phys. Rev. E 90, 030902 (2014).

${ }^{18}$ I. Omelchenko, A. Provata, J. Hizanidis, E. Schöll, and P. Hövel, Phys. Rev. E 91, 022917 (2015).

${ }^{19}$ M. J. Panaggio and D. M. Abrams, Nonlinearity 28, R67 (2015).

${ }^{20}$ G. C. Sethia and A. Sen, Phys. Rev. Lett. 100, 144102 (2008).

${ }^{21}$ C. R. Laing, Chaos 19, 013113 (2009).

${ }^{22}$ L. Schmidt, K. Schönleber, K. Krischer, and V. Garca-Morales, Chaos 24, 013102 (2014).

${ }^{23}$ L. Schmidt and K. Krischer, Phys. Rev. Lett. 114, 034101 (2015).

${ }^{24}$ S. W. Haugland, L. Schmidt, and K. Krischer, Sci. Rep. 5, 16312 (2015).

${ }^{25}$ R. Gopal, V. K. Chandrasekar, A. Venkatesan, and M. Lakshmanan, Phys. Rev. E 89, 052914 (2014).

${ }^{26}$ A. M. Hagerstrom, T. E. Murphy, R. Roy, P. Hövel, I. Omelchenko, and E. Schöll, Nat. Phys. 8, 658 (2012).

${ }^{27}$ M. Tinsley, S. Nkomo, and K. Showalter, Nat. Phys. 8, 662 (2012).

${ }^{28}$ E. A. Martens, S. Thutupalli, A. Fourrière, and O. Hallatschek, Proc. Natl. Acad. Sci. U.S.A. 110, 10563 (2013).

${ }^{29}$ M. Wickramasinghe and I. Z. Kiss, PLoS One 8, e80586 (2013).

${ }^{30}$ M. Wickramasinghe and I. Z. Kiss, Phys. Chem. Chem. Phys. 16, 18360 (2014).

${ }^{31}$ L. Larger, B. Penkovsky, and Y. Maistrenko, Phys. Rev. Lett. 111, 054103 (2013).

${ }^{32}$ K. Schnleber, C. Zensen, A. Heinrich, and K. Krischer, New J. Phys. 16, 063024 (2014).

${ }^{33}$ L. Larger, B. Penkovsky, and Y. Maistrenko, Nat. Commun. 6, 7752 (2015).

${ }^{34}$ A. Zaikin and A. M. Zhabotinsky, Nature 225, 535 (1970).

${ }^{35}$ L. Kuhnert, Nature 319, 393 (1986).

${ }^{36}$ A. F. Taylor, P. Kapetanopoulos, B. J. Whitaker, R. Toth, L. Bull, and M. R. Tinsley, Phys. Rev. Lett. 100, 214101 (2008).

${ }^{37}$ S. Nkomo, M. R. Tinsley, and K. Showalter, Phys. Rev. Lett. 110, 244102 (2013).

${ }^{38}$ The transmitted light intensity $I_{j}$ of each oscillator is measured in gray level, and the projected light intensity $\phi_{j}$ is determined by gray level, where the background intensity $\phi_{0}=140$, which is equal to $1.4 \mathrm{~mW} \mathrm{~cm}^{-2}$.

${ }^{39}$ A. M. Zhabotinsky, F. Bucholtz, A. B. Kiyatkin, and I. R. Epstein, J. Phys. Chem. 97, 7578 (1993).

${ }^{40}$ R. Toth, A. F. Taylor, and M. R. Tinsley, J. Phys. Chem. B 110, 10170 (2006).

${ }^{41}$ S. Nichols and K. Wiesenfeld, Phys. Rev. A 45, 8430 (1992).

${ }^{42}$ M. Silber, L. Fabiny, and K. Wiesenfeld, J. Opt. Soc. Am. B 10, 1121 (1993).

${ }^{43}$ S. H. Strogatz and R. E. Mirollo, Phys. Rev. E 47, 220 (1993).

${ }^{44}$ W. Zou and M. Zhan, SIAM J. Appl. Dyn. Syst. 8, 1324 (2009).

${ }^{45}$ S. Olmi, A. Politi, and A. Torcini, J. Math. Neurosci. 2, 12 (2012).

${ }^{46}$ Z. Zheng, G. Hu, and B. Hu, Phys. Rev. Lett. 81, 5318 (1998). 